\title{
Experiencia de un programa de cesación del tabaquismo en el Instituto Nacional del Tórax
}

\author{
SERGIO BELLO S.***, HAYDÉE CHAMORRO R.*, MARÍA DE LOS ÁNGELES ORELLANA F.*, \\ LORENA CISTERNAS T.* y MAURICIO SALINAS F.*
}

\section{Experience of a smoking cessation program from a Chilean chest hospital}

Background: Tobacco use is one of the main preventable causes of morbidity and mortality in the world. This report presents the experience of the smoking cessation team from the National Thorax Institute (Instituto Nacional del Tórax-Chile). Patients and Method: A clinical series of patients treated between April 2013 and March 2014, with one-year follow-up was studied. Intervention was based on seven group sessions, with a cognitive behavioral viewpoint and pharmacological treatment (mainly varenicline). Follow-up was done through telephone calls at 1, 3, 6 and 12 months. Descriptive statistics and $\chi^{2}$ test were used. Results: Eighty-seven patients were treated, mean age 54 years, 63\% women; 79\% had a pack year index over 20; 28\% had depression and 16\% had COPD. 59\% received varenicline. Self-reported abstinence for 12 months was 37\%. No significant differences between highrisk groups were found. Conclusion: The smoking cessation program done at the National Thorax Institute shows that it is feasible to implement this type of programs in the public health system of Chile with results comparable to those internationally published.

Key words: Smoking cessation; varenicline; cognitive behavioral therapy.

\section{Resumen}

Introducción: El tabaco es uno de los principales factores de morbimortalidad prevenible en el mundo. En este artículo se presenta la experiencia del equipo de tratamiento del tabaquismo en el Instituto Nacional del Tórax (INT). Método: Se analizaron los pacientes tratados entre abril de 2013 y marzo de 2014, con seguimiento a un año. La intervención consistió en 7 sesiones grupales con enfoque cognitivo conductual y terapia farmacológica (principalmente vareniclina). El seguimiento fue telefónico al mes 1, 3, 6 y 12. Se utilizó estadística descriptiva y test de $\chi^{2}$. Resultados: Se sometieron a tratamiento 87 pacientes, edad promedio 54 años, 63\% mujeres; tienen indice paquete año sobre 20 el 79\%, depresión 28\% y Enfermedad Pulmonar Obstructiva Crónica 16\%. El 59\% recibió vareniclina. La abstinencia autoreportada a 12 meses fue de 37\%. No se encontraron diferencias significativas en grupos de riesgo. Conclusión: La experiencia de tratamiento anti-tabaco realizada en el INT muestra que es factible implementar este tipo de programas en el sistema público de salud chileno con resultados comparables a las publicaciones internacionales.

Palabras clave: Tratamiento del tabaquismo; vareniclina; terapia cognitivo-conductual.

\section{Introducción}

El consumo de tabaco mata a la mitad de sus consumidores, constituyéndose en una verdadera epidemia mundial propagada principalmente por los intereses comerciales de las compañías de tabaco. Estas son empresas transnacionales, que cubren con su influencia financiera y política, todo el planeta ${ }^{1}$. Para hacer frente a esta situación de alarma para la salud pública mundial, la Organización Mundial de la Salud (OMS) propuso una estrategia internacional para el control del consumo de tabaco. En el año 2003 se firmó por parte de la mayoría de los países miembros de la

* Programa de Tabaquismo. Instituto Nacional del Tórax. Santiago, Chile.

** Profesor Adjunto, Facultad de Medicina e INTA, Universidad de Chile. 
OMS, el primer tratado de salud pública del mundo, denominado Convenio Marco para el Control del Tabaco $(\mathrm{CMCT})^{2}$, que Chile aprobó como ley de la República en marzo de 2005.

Uno de los puntos de acuerdo del CMCT (artículo 14) es que debe disponerse de terapia antitabáquica para ofrecerla a todos los fumadores. En Chile esto no se ha abordado en la forma de un programa de cobertura nacional, lo que sin duda es un aspecto pendiente por parte del Ministerio de Salud, por lo cual además se está incumpliendo el CMCT.

El Instituto Nacional del Tórax (INT) fue el primer hospital público del país en desarrollar un Programa de Cesación del Tabaquismo. En esta institución se realizan terapias antitabáquicas desde hace más de 20 años, tanto en modalidad individual como grupal. En 1994 publicamos nuestra experiencia inicial ${ }^{3}$, y en el presente artículo queremos dar a conocer la experiencia más reciente, utilizando terapia grupal, que es la forma en que atendemos a la mayoría de los pacientes, por su mejor relación costo-efectividad.

\section{Material y Método}

Es un estudio descriptivo con seguimiento de los pacientes que participaron en terapias grupales desde abril de 2013 a marzo de 2014, período en que efectuó esta terapia en un total de 8 Grupos consecutivos, realizándose el corte evaluativo en abril de 2015. La información obtenida fue ingresada a una base de datos a la cual pudieron acceder sólo los profesionales a cargo del estudio. El protocolo utilizado en los pacientes participantes corresponde a la forma habitual de trabajo del policlínico de tabaquismo del INT, por lo tanto, este artículo comunica una experiencia del "mundo real".

\section{Pacientes}

Los pacientes fueron evaluados en forma individual a través de una entrevista clínica realizada por médico o psicóloga del Programa. En esta entrevista se evalúa:

1. Grado de adicción a través del test de Fagerström.

2. Motivación para dejar de fumar a través del test de Richmond.

3. Estado psico-emocional con el test de Golberg.

4. Etapa del proceso de cambio en que se encuentra.

Después de la evaluación inicial se integraron a terapia grupal, cada uno de ellos conformado por 8-12 pacientes.
El único criterio de exclusión, fue tener 5 o más puntos en el test de Golberg, que hace probable un trastorno depresivo. Dichos pacientes son derivados a salud mental y se les dejó abierta la posibilidad de volver una vez estabilizados de su cuadro psiquiátrico.

\section{Intervención}

La terapia grupal consiste en 7 sesiones una vez a la semana. Cada sesión dura 1,5 a 2 h, en que participan dos miembros del equipo terapéutico, médico y psicóloga. Las enfermeras realizan seguimiento telefónico intra tratamiento (entre una sesión y otra) y con posterioridad al término de las 7 sesiones, al mes 1, 3, 6 y 12, utilizando protocolos pre-establecidos. En forma ocasional se realizó control presencial al detectarse en el seguimiento telefónico sospecha de cuadro depresivo, caídas (lapses) de la abstinencia tabáquica $\mathrm{u}$ otro problema del paciente. Al constatarse telefónicamente una recaída, se le dio al paciente la posibilidad de volver a realizar la terapia grupal, cuando se sintiera motivado y preparado.

Los resultados de la abstinencia tabáquica se obtuvieron por declaración directa de los participantes, ya que no realizamos en nuestra práctica habitual validación biológica. No se dispone en nuestro hospital de medición de cotinina, sí se cuenta con co-oxímetro que nos permite medir $\mathrm{CO}$ (monóxido de carbono) en aire espirado, que lo utilizamos como un incentivo a la cesación durante la terapia.

Se realizó una intervención con un enfoque cognitivo conductual, en que los fumadores participan en un plan integral de tratamiento que incluye las dimensiones educacional, farmacológica, psicológica y social. La descripción cualitativa del proceso terapéutico desde la sesión $1 \mathrm{a}$ la 7 se muestra en el anexo 1 .

En cuanto al uso de medicamentos, hasta agosto de 2013 el hospital no disponía en su arsenal farmacológico de ningún medicamento de apoyo, por lo que cada paciente debía autofinanciarlo, quedando sujeto a la capacidad económica del paciente. Desde septiembre del año 2013 disponemos de Vareniclina para entrega gratuita a los fumadores, que es actualmente la indicación habitual. En algunos pacientes se agrega chicle de nicotina, si puede pagarlos y si la adicción es muy alta (Fagerström $\geq 7$ puntos). En los fumadores que además tienen algún trastorno de salud mental con uso de psicofármacos, se le solicita a su psiquiatra tratante autorización para el uso de Vareniclina. En algunos casos, especialmente en aquellos con antecedentes de depresión en el pasado, se utilizó Bupropión. A todos los pacientes 
que utilizaron medicamentos, se les indicó por un período de dos meses.

\section{Análisis estadístico}

La descripción de variables se realizó mediante media y desviación estándar (DS) para aquellas cuantitativas y frecuencias absolutas y relativas para las cualitativas.

Para la comparación entre variables categóricas se utilizó prueba de chi cuadrado $\left(\chi^{2}\right)$. Se utilizó un nivel de significación de 0,05 a dos colas.

\section{Resultados}

La muestra estuvo constituida por 87 pacientes, incorporados a terapia grupal en 8 grupos sucesivos desde marzo de 2013 a abril de 2014.

Las características principales del grupo de fumadores se observan en la Tabla 1. El 63\% eran mujeres, con promedio de edad de 54,4 años. La carga de consumo de tabaco evaluada a través del índice paquete-año (IPA) fue 37 (valor promedio), en que el $79 \%$ de ellos tenían un IPA igual o mayor a 20. Dos tercios de los pacientes tenían patologías asociadas, siendo las más prevalentes los trastornos depresivos con $28 \%$ y EPOC $16 \%$. En cuanto al grado de adicción medido por el test de Fagerström el promedio fue de 5,73 puntos y en que el $43 \%$ tenían 7 o más puntos.

Se evaluó el estado psico-emocional de los participantes con el test de Golberg: su promedio fue de 2,5 puntos. Sólo hubo dos fumadores con 5 o más puntos que fueron incluidos dada su alta motivación y disposición al cambio (se encontraban en etapa de preparación).

La motivación fue evaluada con el test de Richmond, que mostró un $74,7 \%$ con alta motivación y sólo un participante con baja motivación.

La mayoría de los pacientes, de acuerdo a las etapas del proceso de cambio de Prochaska y Di Clemente, se encontraba en etapa de contemplación $(65,5 \%)$, el $19,5 \%$ en preparación y un $3,5 \%$ en acción, al ingresar a la terapia grupal. Esto último se produce porque existe, a veces, un desfase entre la evaluación inicial y el comienzo de la terapia grupal.

En cuanto a la asistencia a las sesiones del tratamiento, se observa un promedio de asistencia a las 7 sesiones de $84 \%$.

El 72\% de los pacientes recibió apoyo farmacológico, principalmente Vareniclina, otros recibieron chicle de nicotina, unos pocos bupropión y algunas mezclas, como se observa en la Tabla 2. La indicación de uso de medicamentos fue por 8 semanas, especialmente con Vareniclina, que fue el único aportado por el hospital a los pacientes.
La abstinencia lograda en los 87 pacientes después de 1 año de seguimiento fue de 36,8\%. En la Figura 1 observamos la abstinencia en cada uno de los períodos en que se realizó la evaluación (1, 3, 6 y 12 meses de seguimiento).

Se analizaron algunos parámetros de la muestra con respecto a la abstinencia de tabaco, al completar un año de seguimiento.

Si separamos a los pacientes que recibieron medicamentos de los que no los recibieron, podemos ver en la Figura 2 las diferencias encontradas en abstinencia, las que no fueron significativas $\left(\mathrm{p}=0,68 ; \chi^{2}\right)$.

Se analizaron los resultados según género, que no mostró diferencias en la abstinencia de mujeres versus hombres $\left(\mathrm{p}=0,14 ; \chi^{2}\right)$.

Tabla 1. Características de la muestra de 87 fumadores incorporados a terapia grupal de tabaquismo

\begin{tabular}{|lc|}
\hline & Media (DS) \\
Edad (años) & $54,4(11,5)$ \\
Índice paquetes año & $36,8(21,5)$ \\
Test de Fagerström (puntos) & $5,7(2,3)$ \\
Test de Golberg (puntos) & $2,6(2,6)$ \\
& $\mathbf{n}(\mathbf{\%})$ \\
Hombres & $32(36,7)$ \\
Patologías asociadas & \\
EPOC & $14(16,1)$ \\
Cardiopatía coronaria & $7(8,1)$ \\
Trastornos depresivos & $24(27,6)$ \\
Otras & $30(34,4)$ \\
Sin otra patología & $29(33,3)$ \\
Motivación & \\
Alta & $65(74,7)$ \\
Media & $16(18,3)$ \\
Baja & $1(1,2)$ \\
Etapa de cambio & \\
Contemplación & $57(65,5)$ \\
Preparación & $17(19,5)$ \\
Acción & $3(3,5)$ \\
\hline
\end{tabular}

Tabla 2. Medicamentos utilizados en terapia grupal cesación tabaquismo

\begin{tabular}{|lcc|}
\hline Medicamento & n & \% \\
\hline Vareniclina & 37 & 59 \\
Chicle de nicotina & 13 & 21 \\
\hline Bupropión & 7 & 11 \\
Vareniclina + chicle de nicotina & 4 & 6 \\
Bupropión + chicle de nicotina & 2 & 3 \\
Total & $\mathbf{6 3}$ & $\mathbf{1 0 0}$ \\
\hline
\end{tabular}




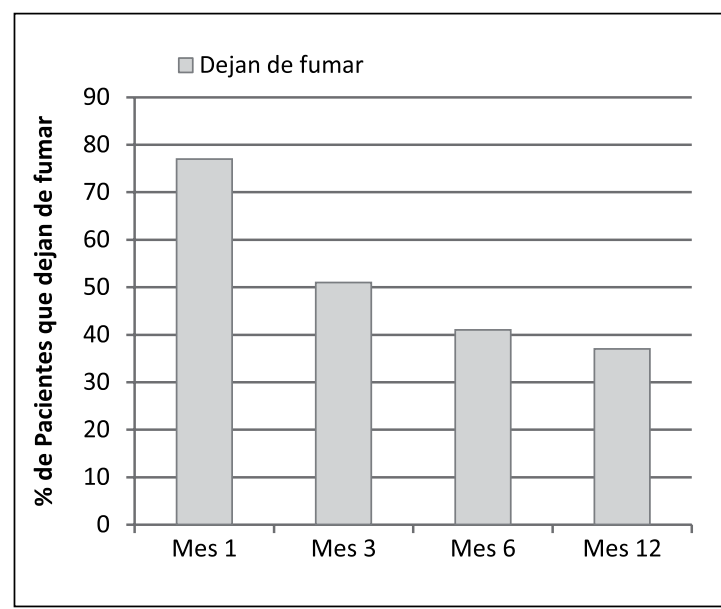

Figura 1. Porcentaje de pacientes que dejan de fumar al mes $1,3,6$ y 12 .
También evaluamos lo que ocurría con las comorbilidades y el resultado de la terapia, especialmente los pacientes con EPOC y los con trastornos depresivos (Figura 3), en los que tampoco hay diferencias estadísticamente significativas $(\mathrm{p}=0,26$ y 0,36 respectivamente).

Se analizó si habían diferencias en los resultados por otras variables: IPA más de 20 o menos de 20 y puntaje de la escala de Fagerström mayor o menor de 7, no encontrando diferencias significativas ( $\mathrm{p}=0,15$ y 0,054 respectivamente).

En cuanto a efectos secundarios de los medicamentos usados, lo único destacable fue la aparición de náuseas en los usuarios de vareniclina en un $17 \%$ ( $7 / 41)$, y que en ninguno de los casos condicionó el retiro del fármaco.

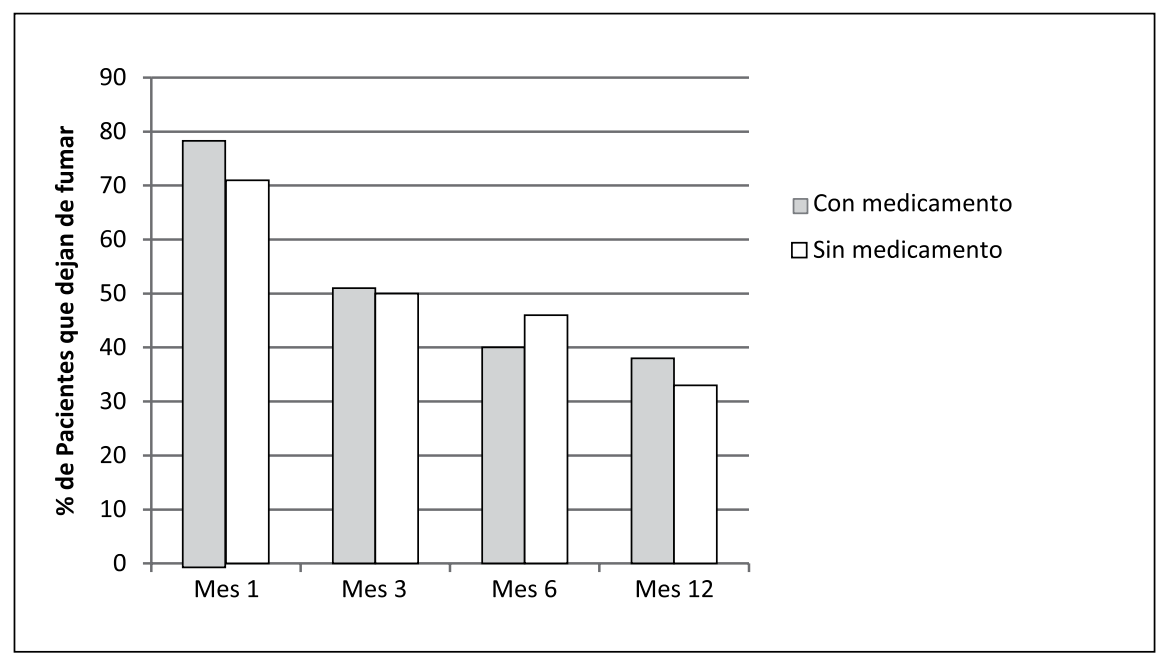

Figura 2. Porcentaje de pacientes que dejan de fumar según uso de medicamento.

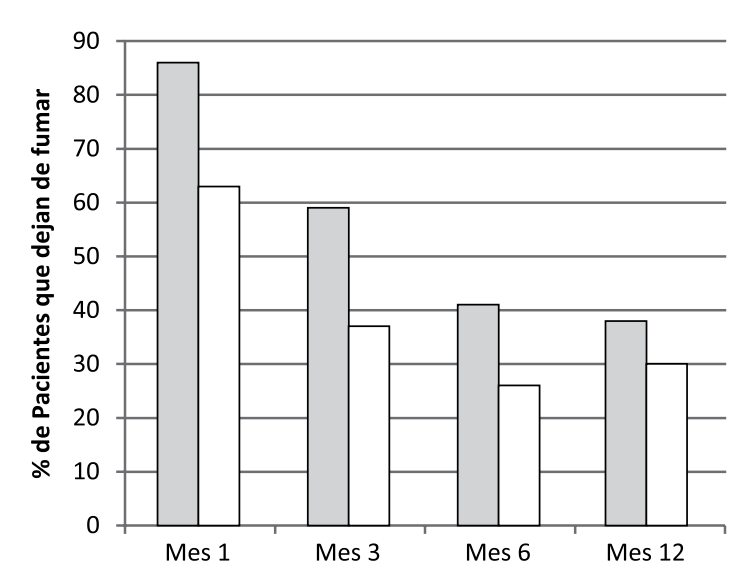

$\square$ Sin trastornos depresivos

$\square$ Con trastornos depresivos
Figura 3. Porcentaje de pacientes que dejan de fumar según la presencia o ausencia de trastornos depresivos. 


\section{Discusión}

Es necesario considerar que la experiencia mostrada corresponde al programa de atención habitual en nuestro hospital, es decir, es una experiencia del "mundo real", en que la única restricción de ingreso, como ya se dijo, es un test de Golberg con 5 o más puntos y en que todos los procedimientos de ingreso, tipo de terapia y seguimiento son los que realizamos en la práctica clínica diaria.

Como ya fue descrito en resultados, estudiamos un grupo de pacientes, en su mayoría mujeres, edad promedio de 54 años, con una gran carga tabáquica y en que dos tercios presenta patologías asociadas, especialmente trastornos depresivos y EPOC, lo que es frecuente en fumadores de larga data ${ }^{4}$. La mayoría tenía un grado de adicción moderado a severo (de acuerdo al test de Fagerström) y el 65,5\% se encontraba en etapa de contemplación, lo que lo hace un grupo especialmente difícil para obtener la abstinencia tabáquica. Esto se contrarresta en parte con que casi el $75 \%$ de los participantes estaban con un alto grado de motivación para el cese. Estos dos factores (grado de adicción y motivación) se considera que contribuyen de manera importante en los resultados de las terapias ${ }^{5}$.

En cuanto a la asistencia a las sesiones grupales ( $84 \%$ en promedio) consideramos que fue bastante buena. Se sabe que la asistencia a la terapia y el tiempo total de contacto entre terapeutaspacientes es un factor importante en relación a los resultados finales ${ }^{5}$. La participación en cada etapa del tratamiento es fundamental para "vivir" el proceso, tanto en relación a los objetivos terapéuticos establecidos para cada sesión, como al apoyo mutuo que se logra entre los miembros del grupo.

Se destaca en la descripción del proceso de la terapia grupal (Anexo 1) los principios de aceptación incondicional de los pacientes, actitud no juzgadora por parte del equipo terapéutico, con escucha activa, apoyo al lenguaje de cambio, enfrentamiento del balance decisional (pro y contra de dejar de fumar), expresión de comprensión, etc. Es decir, utilizamos los principios y estrategias de la entrevista motivacional (expresar empatía, desarrollar las discrepancias, lidiar con la resistencia y apoyar la autoeficacia), metodología que se está utilizando en diversos países y escenarios ${ }^{6}$ como enfrentamiento a los fumadores.

Los resultados en cuanto a la cesación tabáquica son bastante satisfactorios (41 y $36,8 \%$ a los 6 y 12 meses respectivamente), comparables a las mejores experiencias tanto nacionales como internacionales ${ }^{7,8}$. Si bien no tenemos validación biológica de los resultados, de acuerdo a experiencias publicadas, hay coincidencia entre las respuestas de los fumadores y las pruebas biológicas de verificación 9 . Consideramos que en el logro de estos buenos resultados influyó de manera fundamental el seguimiento telefónico realizado por enfermera, lo que fue explícitamente reconocido por los pacientes, corroborando otras experiencias comunicadas ${ }^{5,10}$.

En los pacientes que utilizaron fármacos no se observaron mejores resultados que en aquellos que no los usaron. Esto no se condice con lo publicado ${ }^{5,11}$ ya que hay consenso internacional que los medicamentos contribuyen de manera significativa al éxito de las terapias, con la excepción de la experiencia del Lung Health Study ${ }^{12}$ y en que la explicación para tal resultado estaría dada por la cantidad (12 sesiones) y buena calidad de la intervención psico-social a la que fueron sometidos todos los pacientes que participaron en ese estudio. Frente al hecho de no observar efecto de la medicación usada se debe considerar que en nuestra serie utilizamos los medicamentos, y específicamente la Vareniclina, por sólo dos meses y no tres como recomienda el fabricante y las publicaciones que avalan su uso. Por otra parte, el número de sujetos que usaron fármacos es pequeño, lo cual podría influir en los resultados. Cabe destacar que en otro estudio nacional desarrollado en un centro privado de Santiago ${ }^{7}$, tampoco hubo un efecto del uso de apoyo farmacológico sobre la abstinencia lograda.

En cuanto a las diferencias que podrían darse entre los distintos medicamentos utilizados, no es posible extraer algunas conclusiones ya que la mayoría usó vareniclina (por estar disponible en el hospital), y pocos bupropión o sustitutos de nicotina, siendo subgrupos muy pequeños y que no permiten hacer comparaciones de validez estadística.

En nuestro grupo de fumadores encontramos que no hubo diferencias por género en los resultados obtenidos, lo que está de acuerdo a la experiencia nacional e internacional ${ }^{7,13}$.

En cuanto a los fumadores con EPOC, no encontramos diferencias significativas en los resultados de la abstinencia con los fumadores sin esa patología, lo que corrobora diversas publicaciones que avalan que los fumadores con comorbilidad no tienen peor pronóstico en la cesación que aquellos "sanos", aunque requieren de tratamientos más extensos en tiempo de dedicación, uso de fármacos asociados y por períodos más prolongados ${ }^{14,15}$.

Por otra parte, los pacientes con antecedentes 
de trastornos depresivos obtuvieran porcentajes de abstinencia similares a la de los fumadores sin problemas de salud mental. Está descrito en diversas publicaciones ${ }^{5,7}$ que el antecedente de depresión puede ser un factor independiente de mal pronóstico, lo que no ocurrió en nuestra experiencia. Sin embargo, en los porcentajes que se muestran en la Figura 3 se observa una tendencia a peores resultados en el grupo con depresión. Probablemente esta diferencia no alcanzó significación estadística $(\mathrm{p}=0,36)$ por un problema de tamaño de la muestra. La dificultad para conseguir la abstinencia es mayor en estos pacientes, aunque con el tratamiento adecuado se pueden conseguir cifras muy similares a la población general, requiriendo de un control y un soporte más estrecho durante el tratamiento ${ }^{16}$.

En resumen, podemos decir que en un grupo de fumadores altamente adictos pero bien motivados, con presencia importante de comorbilidades, al aplicar terapia grupal utilizando los principios de la entrevista motivacional más apoyo de fármacos se logran buenos resultados. Por otra parte, el trabajo en equipo de médico, psicóloga y enfermera contribuyen al desarrollo de una adecuada práctica clínica, que favorecen estos buenos resultados y el buen uso de los escasos recursos de un hospital público.

\section{Anexo 1. Descripción del proceso terapéutico durante la terapia grupal}

Los participantes se someten a un plan integral de tratamiento que considera las dimensiones educacional, psicológica y social, con un enfoque cognitivo conductual

Enmarcados en los principios de la Entrevista Motivacional, que a través de la empatía y el desarrollo de las discrepancias logra suscitar en cada fumador la mejor motivación para el cambio, los terapeutas entregan a los fumadores el protagonismo en el proceso de lograr y mantener la abstinencia. Se establece así, una relación horizontal terapeutasgrupo, creándose un clima caracterizado por calidez, aceptación incondicional, alta motivación, apertura y valoración de la experiencia de los otros. En este contexto, cada participante es a la vez paciente y terapeuta del grupo, ya que cada vivencia compartida en el grupo adquiere un valor formativo

Las tres primeras sesiones, correspondientes al período de preparación para dejar de fumar, se enfocan en construir un clima de confianza básica, conocer las características de la adicción y mostrar los daños producidos por el tabaquismo, así como dar herramientas para el manejo de las conductas relacionadas con la adicción, identificando las condicionantes del fumar que permiten caracterizar los grupos

En cada grupo de tratamiento se evidenció alta motivación para abandonar el tabaquismo, apareciendo la salud, la calidad de vida, la relación familiar, el ejemplo para los hijos y el logro de libertad y crecimiento personal como los motivos más frecuentes que fundamentan la decisión para participar en el tratamiento

Entre las sesiones 3 y 4 se fija el “día D” (primer día sin fumar), desde donde la terapia se enfoca en el manejo del síndrome de privación, en la mantención de la abstinencia, la prevención de recaídas y los cambios hacia una conducta definitiva de no fumador. El logro de estos objetivos se basa en técnicas de fortalecimiento de la autoconfianza, autoeficacia, refuerzo individual y social, técnicas de manejo de tensiones (ansiedad y craving) y apoyo farmacológico

En cada grupo se trabajan las vivencias que surgen de poner fecha al primer día sin fumar. Al fijar el día D se producen sentimientos ambivalentes, con reacciones de angustia, temores paralizantes y dudas respecto a la capacidad para lograr la abstinencia. Sin embargo, habitualmente prima la expectación positiva y la motivación para enfrentar el desafío

Las sesiones 5 y 6 se centran en valorar los beneficios asociados al dejar de fumar y a transformar un compromiso formal en cambio conductual duradero previniendo recaídas. Para lograr este objetivo, sesión a sesión se les van entregando las herramientas necesarias: técnicas de relajación, estrategias diversas para enfrentar las tentaciones, entrenamiento en asertividad, con refuerzo individual y social

Las herramientas de apoyo para enfrentar el síndrome de privación y la etapa de abstinencia son recibidas positivamente en todos los grupos. Las técnicas empleadas permiten a los participantes ponerse en contacto consigo mismo, explorar ganancias secundarias al remitir la conducta problema, identificar y controlar conductas automáticas, reforzar la decisión de abstinencia, logrando no sólo la meta prevista, sino además objetivos secundarios de fortalecimiento de autoestima y autoeficacia, que instan a tomar otras decisiones en pro de enriquecer y mejorar sus estilos de vida

La potencia del refuerzo social al interior del grupo queda de manifiesto en las últimas sesiones, al fomentar la motivación de aquellos fumadores que evidencian mayor dificultad para lograr la abstinencia. La sesión 7 se consolida el apoyo grupal, se evalúa el tratamiento y se proyecta la abstinencia en el tiempo

Los participantes coinciden en asignar al apoyo grupal la categoría de variable muy relevante en la consecución del objetivo de dejar de fumar, y cómo el logro de esta meta tiene un impacto significativo en sus vidas, al eliminar un factor de riesgo para su salud, propiciar la extensión de la motivación de logro hacia otras áreas del desarrollo personal y mejorar la sobrevida y calidad de vida para sí mismos y de quienes les rodean 


\section{Bibliografía}

1.- ORGANIZACIÓN MUNDIAL DE LA SALUD. http:// www.who.int/tobacco/industry/es/ (Consultado el 15 de marzo de 2015).

2.- ORGANIZACIÓN MUNDIAL DE LA SALUD, GINEBRA, SUIZA. Convenio Marco de la OMS para el control del Tabaco. OMS 2003, reimpresión actualizada, 2004, 2005. ISBN 9243591010

3.- BELLO S, CORVALÁN M P. Tratamiento del tabaquismo: Experiencia del Instituto Nacional de Enfermedades Respiratorias. Rev Chil Enferm Respir 1994; 10: 13642.

4.- JIMÉNEZ C A, MAYAYO M, CISERO A, AMOR N, RUIZ J J, FERNÁNDEZ M C, et al. Resultados asistenciales de una unidad especializada en tabaquismo. Arch Bronconeumol 2009; 45: 540-4.

5.- FIORE M C, JAEN C R, BAKER T R, BAILEY W C, BENOWITZ N, CURRY S, et al. Treating tobacco use and dependence. 2008 update. Clinical Practice Guideline. Rockville. US Department of Health and Human Services. Public Health Service. 2008.

6.- LÜHNING S, BULJUBASICH D. Entrevista motivacional: la nueva estrella de la película. Prev Tab 2014; 16: 7-11.

7.- PEÑA P, ZAGOLÍN M, ACUÑA M, NAVARRETE S, BUSTAMANTE P, SUÁREZ C, et al. Resultados de un programa multidisciplinario para el control del hábito tabáquico. Rev Med Chile 2013; 141: 345-52.

8.- IZQUIERDO MONGE M D, SÁNCHEZ PÁEZ Y, RODRÍGUEZ VÁZQUEZ S, VELASCO ESPINOSA I, SEGURA BALBUENA M, ORTEGA CALVO M. Evaluación de la intervención grupal avanzada en una muestra de fumadores. Prev Tab 2014; 16: 115-20.

9.- BARRUECO M, JIMÉNEZ RUIZ C, PALOMO L, TO-
RRECILLA M, ROMERO P, RIESCO J A. Veracidad de la respuesta de los fumadores sobre su abstinencia en las consultas de deshabituación tabáquica. Arch Bronconeumol 2005; 41: 135-40.

10.- STEAD L F, HARTMANN-BOYCE J, PERERA R, LANCASTER T. Telephone counselling for smoking cessation. Cochrane Database of Systematic Reviews 2013, Issue 8. Art. $\mathrm{N}^{\mathrm{o}}$ : CD002850. DOI: 10.1002/14651858.CD002850.pub 3.

11.- HARTMANN-BOYCE J, STEAD L F, CAHILL K, LANCASTER T. Efficacy of interventions to combat tobacco addiction: Cochrane update of 2013 reviews. Addiction. Cochrane Updates. doi:10.1111/add.12633.

12.- ANTHONISEN N R, CONNETT J E, KILEY J P, ALTOSE M D, BAILEY W C, BUIST A S, et al. Effects of smoking intervention and the use of an inhaled anticholinergic bronchodilator on the rate of decline of $\mathrm{FEV}_{1}$ : The Lung Health Study. JAMA 1994; 272: 1497-505.

13.- DE HIGES MARTÍNEZ E B, RAMOS PINEDO A. Diferencias de género en el abandono del tabaco. Prev Tab 2013; 15: 114-20.

14.- JIMÉNEZ-RUIZ C, RIESCO MIRANDA J A, ALTET GÓMEZ N, LORZA BLASCO J J, SIGNES-COSTA MIÑANA J, SOLANO REINA S, et al. Tratamiento del tabaquismo en fumadores con enfermedad pulmonar obstructiva crónica. Arch Bronconeumol 2013; 49: 35463.

15.- BELLO S, CHAMORRO H, BARRIENTOS A. Tratamiento del tabaquismo en pacientes con enfermedad pulmonar obstructiva crónica. Rev Chil Enferm Respir 2013; 29: 24-30.

16.- HISADO DÍAZ M D. Tratamiento del tabaquismo en pacientes con patología depresiva. Revisión. Prev Tab 2011; 13: 160-6.

Correspondencia a:

Dr. Sergio Bello S.

Instituto Nacional del Tórax

Avda. José M. Infante 717, Providencia.

Santiago, Chile.

Email: sergio.bello@torax.cl 